IMA Journal of Mathematical Control and Information Page 1 of 22 doi:10.1093/imamci/dnnxxx

\title{
Structure-preserving discretization and control of a 2D vibro-acoustic tube
}

\author{
YONGXIN WU, YANN LE GORREC \\ FEMTO-ST, Univ. Bourgogne Franche-Comté, CNRS, 24 rue Savary, F-25000 Besançon, \\ France. \\ HECTOR RAMIREZ \\ Department of Electronic Engineering, Universidad Tecnica Federico Santa Maria, Avenida \\ Espana 1680, Valparaiso, Chile \\ LAURENT LEFÈVRE \\ Univ. Grenoble Alpes, Grenoble INP, LCIS, 26000 Valence, France
}

\begin{abstract}
This paper deals with the structure-preserving discretization and control of a 2D vibro-acoustic tube using the port-Hamiltonian framework. A discretization scheme is proposed and a set of precise weighting functions are given in order to obtain a structure preserving finite dimensional port- Hamiltonian approximation of the 2D vibro-acoustic system. Using the closed-loop structural invariants of the approximated system an energy-Casmir controller is derived. The performance of the proposed discretizetion scheme and the controller is shown by means of numerical simulations.
\end{abstract}

Keywords: Structure-preserving discretization, Control by interconnection, Vibro-acoustic system, Casmir invariant

\section{Introduction}

Based on the energy and a structured representation of the power flows and dissipation in the system, the port-Hamiltonian framework is particularly suited to describe the complex behavior of multi-physical systems [Duindam et al., 2009]. The port-Hamiltonian approach has been generalized to infinitedimensional systems described by partial differential equations (PDEs) in [van der Schaft and Maschke, 2002, Le Gorrec et al., 2005]. From an application point of view, reduction of vibrations has drawn attention in both academic and industrial areas. Modeling and control of the vibro-acoustic systems have been investigated for different applications [Gardonio, 2002, Durand et al., 2008] in the last few years. The key point in these systems is the wave propagation process which can be modeled in a straightforward manner with the port-Hamiltonian framework. In [Trenchant et al., 2015], a port-Hamiltonian formulation of the $2 \mathrm{D}$ wave propagation has been proposed for a vibro-acoustic system on a rectangular spatial domain.

With the aim of simulating the system in a physically consist way and furthermore the control design and implementation purpose, the numerical discretization scheme should preserve the Hamiltonian structure and the passivity of the original infinite dimensional system on the derived finite dimensional approximation. Research on structure preserving discretization of port-Hamiltonian systems has drawn the attention of researchers in the last years and several methods, such as the ones based on mixed finite elements [Golo et al., 2004, Baaiu et al., 2009], pseudo-spectral [Moulla et al., 2011, Vu et al., 2017], and finite volume have been proposed in [Kotyczka, 2016]. Recently an approach based on discrete exterior geometry has been proposed in [Seslija et al., 2012]. The general idea of these methods is to discretize the energy and co-energy variables using distinct low-order basis functions such that the equa- 
tions was exactly satisfied in these finite dimensional spaces. The mixed Galerkin approach has been proposed to discretize the weak formulation of the Stokes-Dirac Structure in [Kotyczka et al., 2018].

In the 2D case, the authors in [Trenchant et al., 2017a, Trenchant et al., 2018a,b] have proposed to adopt the finite difference method to discretize the 2D port-Hamiltonian system on staggered grids. The finite volume method is employed to discretize 2D linear and non linear port-Hamiltonian systems in [Serhani et al., 2018]. The generalization of the discrete exterior geometry approach to the 2D case has been done in [Kotyczka and Maschke, 2017] on $n$-complexes. The mixed Galerkin discretization applied on the weak formulation of 2D port-Hamiltonian system has been proposed in[Kotyczka et al., 2018]. The partitioned finite element method has been introduced in [Cardoso-Ribeiro et al., 2018] and also applied on the 2D plate models in [Brugnoli et al., 2019a,b].

A preliminary study of the structure preserving discretization of a $2 \mathrm{D}$ vibro-acoustic system using a mixed finite element method has been proposed in [Wu et al., 2015]. In the present paper, we propose an exact discretized finite dimensional model with a precise choice of basis functions that satisfy the compatibility conditions associated with the discretization. Furthermore, an explicit finite dimensional input-output system is derived when considering the physical input and boundary conditions of 2D actuated vibro-acoustic system. In order to reduce/attenuate the wave propagation, an energy based control method based on closed-loop structural invariants similar to the on proposed in [Trenchant et al., 2017b] is investigated. By using this method, we can shape the energy function in the closed loop system to the desired one with a specific conservative boundary interconnection.

This paper is structured as follows. The infinite dimensional port-Hamiltonian system is recalled in Section 2 and then applied on the 2-D vibro-acoustic system which is obtained from the 3-D model with a geometry reduction [Vu et al., 2019]. Section 3 presents the passivity and structure preserving discretization of the 2-D vibro-acoustic system with a specific choice of the weighting functions and furthermore, the control oriented explicit finite dimensional port-Hamiltonian system is derived. In Section 4, an energy based control is designed using the discretized model using the energy-Casimir method. The numerical simulation results are shown in Section5 to illustrate the effectiveness of the proposed discretization scheme and passive controller. At last, we conclude this paper by Section 6 and then give some interest perspectives.

\section{Port-Hamiltonian formulation of a 2-D vibro-acoustic tube}

\subsection{Infinite dimensional port-Hamiltonian system}

The PDE representation of a port-Hamiltonian system, written in terms of exterior differential calculus on a n-dimensional spatial domain $\mathscr{Z}$ can be decomposed in structure, dynamics and constitutive equation [van der Schaft and Maschke, 2002].

$$
\begin{aligned}
& {\left[\begin{array}{l}
f^{p} \\
f^{q}
\end{array}\right]=\left[\begin{array}{cc}
0 & (-1)^{r} \mathrm{~d} \\
\mathrm{~d} & 0
\end{array}\right]\left[\begin{array}{l}
e^{p} \\
e^{q}
\end{array}\right] \quad \text { Structure }} \\
& {\left[\begin{array}{l}
f^{p} \\
f^{q}
\end{array}\right]=\left[\begin{array}{l}
-\frac{\partial \alpha^{p}}{\partial t} \\
-\frac{\partial \alpha^{q}}{\partial t}
\end{array}\right] \quad \text { Dynamics }} \\
& {\left[\begin{array}{l}
e^{p} \\
e^{q}
\end{array}\right]=\left[\begin{array}{l}
\delta_{\alpha^{p}} H \\
\delta_{\alpha^{q}} H
\end{array}\right] \quad \text { Constitutive Equations }}
\end{aligned}
$$

Denote $\mathscr{Z}$ the $n$-dimensional spatial domain. The state space $X$ is defined as $X:=\Omega^{n_{p}}(\mathscr{Z}) \times \Omega^{n_{q}}(\mathscr{Z})$ with $n_{p}+n_{q}=n+1, r=n_{p} n_{q}+1$ and $\Omega^{n_{p}}$ is the space of $n_{p}$ forms. An element of the space $X$ is 
denoted by $x=\left[\begin{array}{ll}\alpha^{p} & \alpha^{q}\end{array}\right]^{T}$ with state variables $\alpha^{p} \in \Omega^{n_{p}}(\mathscr{Z})$ and $\alpha^{q} \in \Omega^{n_{q}}(\mathscr{Z})$. The flow variables $f^{p}=-\partial_{t} \alpha^{p} \in \Omega^{n_{p}}(\mathscr{Z})^{1}$ and $f^{q}=-\partial_{t} \alpha^{q} \in \Omega^{n_{q}}(\mathscr{Z})$ define the vector of flows $f=\left[f^{p} \quad f^{q}\right]^{T} \in \mathscr{F}:=$ $\Omega^{n_{p}}(\mathscr{Z}) \times \Omega^{n_{p}}(\mathscr{Z})$. The effort variable $e^{p}=\delta_{\alpha^{p}} H \in \Omega^{n-n_{p}}(\mathscr{Z})$ and $e^{q}=\delta_{\alpha^{q}} H \in \Omega^{n-n_{q}}(\mathscr{Z})$ define the vector of efforts $e=\left[\begin{array}{ll}e^{p} & e^{q}\end{array}\right]^{T} \in \mathscr{E}:=\Omega^{n-n_{p}}(\mathscr{Z}) \times \Omega^{n-n_{q}}(\mathscr{Z})$. Flows and efforts represent dual, power-conjugated port variables. The exterior derivative $\mathrm{d}: \Omega^{n-1}(\mathscr{Z}) \mapsto \Omega^{n}(\mathscr{Z})$ can be seen as an unifying differential operator in exterior calculus in order to present the spatial derivative in the domain. $\delta_{\alpha^{p}} H$ and $\delta_{\alpha^{q}} H$ are the variational derivatives of the energy or Hamiltonian functional $H=\int_{\mathscr{Z}} \mathscr{H}$ with the Hamiltonian density $\mathscr{H}: \Omega^{n_{p}}(\mathscr{Z}) \times \Omega^{n_{q}}(\mathscr{Z}) \times \mathscr{Z} \mapsto \Omega^{n}(\mathscr{Z})$. The boundary variables of the PH formulation (1) are defined by

$$
\left[\begin{array}{l}
f^{B} \\
e^{B}
\end{array}\right]=\left[\begin{array}{cc}
1 & 0 \\
0 & -(-1)^{n-n_{q}}
\end{array}\right]\left[\begin{array}{l}
\left.e^{p}\right|_{\partial \mathscr{Z}} \\
\left.e^{q}\right|_{\partial \mathscr{Z}}
\end{array}\right] \quad \text { Boundary Variable }
$$

with $\partial \mathscr{Z}$ the $n-1$ dimensional boundary of the spatial domain $\mathscr{Z}$. The space of all admissible flows and efforts satisfying (1), (4) represents a Stokes-Dirac structure [van der Schaft and Maschke, 2002], with respect to the scalar pairing

$$
\int_{\mathscr{Z}} e^{q} \wedge f^{q}+\int_{\mathscr{Z}} e^{p} \wedge f^{p}+\int_{\partial \mathscr{Z}} e^{B} \wedge f^{B} .
$$

\subsection{The $2 D$ vibro-acoustic tube}

We consider a cylindrical tube system presented in [David et al., 2010], in which an acoustic wave evolves without energy loss. The source of the acoustic wave is produced by a loudspeaker on one side of tube, and an anechoic chamber avoids reflections of the wave at the other side of the tube. We consider an axial symmetry for this system as in Figure 1. This allows to reduce the system from 3D coordinates $(x, y, \phi)$ to $2 \mathrm{D}$ coordinates $(x, r)$ where $x \in[0, L], r \in[0, R], L, R$ are the length and radius of the tube respectively, in a power preserving way by a simple change of variables (explicited later).

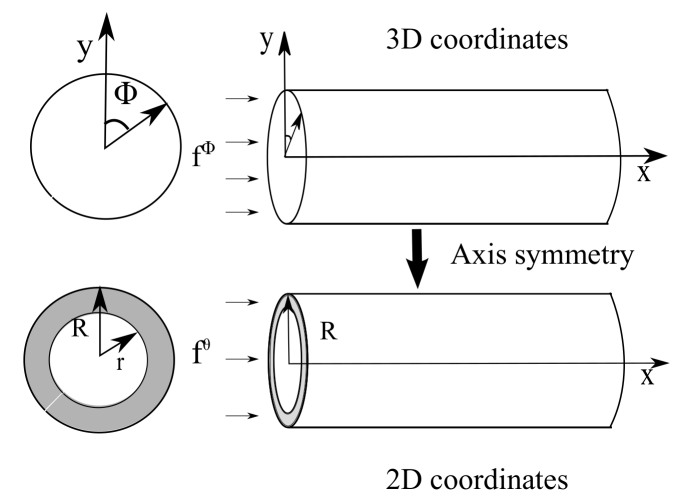

FIG. 1. Reduction from 3D to 2D model by axisymmetry

The 2D infinite dimensional vibro-acoustic system is written as port Hamiltonian system: $\mathscr{Z}$ is a 2-dimensional smooth mainfold with 1-dimensional smooth boundary $\partial \mathscr{Z}$, i.e. $n=2$. The state space

${ }^{1}$ Denote the operator $\frac{\partial}{\partial t}$ by $\partial_{t}$ and $\frac{\partial}{\partial x}$ by $\partial_{x}$ for the sake of simplification. 
$X$ is defined as $X:=\Omega^{2}(\mathscr{Z}) \times \Omega^{1}(\mathscr{Z})$ with $n_{p}=2$ and $n_{q}=1$. The element of the space $X$ is denoted by $x=[\theta, \Gamma]^{T}$ with $\theta$ the kinetic momentum (2-form), and $\Gamma$ the volumetric expansion (one-form). The spaces $\mathscr{F}, \mathscr{E}$ are defined as $\mathscr{F}:=\Omega^{2}(\mathscr{Z}) \times \Omega^{1}(\mathscr{Z})$, and $\mathscr{E}:=\Omega^{0}(\mathscr{Z}) \times \Omega^{1}(\mathscr{Z})$. The elements $f^{x} \in \mathscr{F}, e^{x} \in \mathscr{E}$ are given as $f^{x}=-[\dot{\theta}, \dot{\Gamma}]^{T}$ and $e^{x}=[v, P]^{T}$ where $v$ is the velocity (0-form) and $P$ is the pressure (one-form). The flow variable $f^{\theta}$ (2-form) of the 2D model is obtained by flow $f^{\Phi}$ (3-form) of the 3D model with $f^{\theta}=2 \pi r f^{\Phi}$ as in Figure 1 .

The total energy is given by:

$$
H=\frac{1}{2} \int_{\mathscr{Z}} \theta \wedge \frac{* \theta}{\rho_{0}}+\Gamma \wedge \frac{* \Gamma}{\chi_{s}}
$$

with $*$ the Hodge star operator which converts any $k$-form $w$ on a $n$-dimensional spatial domain $\mathscr{Z}$ to an $(n-k)$-form $* w$. Here $\rho_{0}$ is the air mass density, $\chi_{s}$ is the adiabatic compressibility coefficient.

The port Hamiltonian representation of the vibro-acoustic system defined on a $2 \mathrm{D}$ spatial domain $\mathscr{Z}=(x, y) \subset \mathbb{R}^{2}$ is:

$$
\begin{aligned}
{\left[\begin{array}{l}
f^{\theta} \\
f^{\Gamma}
\end{array}\right] } & =\left[\begin{array}{cc}
0 & -\mathrm{d} \\
\mathrm{d} & 0
\end{array}\right]\left[\begin{array}{l}
e^{v} \\
e^{p}
\end{array}\right] & & \text { Structure } \\
{\left[\begin{array}{l}
f^{\theta} \\
f^{\Gamma}
\end{array}\right] } & =\left[\begin{array}{c}
-\dot{\theta} \\
-\dot{\Gamma}
\end{array}\right] & & \text { Dynamics } \\
{\left[\begin{array}{l}
e^{v} \\
e^{p}
\end{array}\right] } & =\left[\begin{array}{c}
\delta_{\theta} H \\
\delta_{\Gamma} H
\end{array}\right] & & \text { Constitutive Equations, } H \text { being defined by (6) } \\
{\left[\begin{array}{l}
f^{B} \\
e^{B}
\end{array}\right] } & =\left[\begin{array}{ll}
1 & 0 \\
0 & 1
\end{array}\right]\left[\begin{array}{l}
\left.e^{v}\right|_{\partial \mathscr{Z}} \\
\left.e^{p}\right|_{\partial \mathscr{Z}}
\end{array}\right] & & \text { Boundary Variables }
\end{aligned}
$$

\section{Discretization of the 2D vibro-acoustic system}

In this section, we propose a structure preserving mixed finite elements discretization method, to derive a finite dimensional approximation of (7) and (10).

\subsection{Approximation of the Dirac structure}

Taking the spatial domain geometry of the system into account, we propose to use infinitesimal square grid elements instead of triangular one found in the literature [Golo et al., 2004]. The square grid element $\mathscr{Z}_{a b c d}$ is defined by four vertices $a, b, c, d$. The edges of the square are defined by $a b, b c, c d, d a$ and the facet by $a b c d$.

We approximate the flow and effort variables on the infinitesimal square grids by using the space dependent weighting forms. The objective is to separate flow and effort variables $f(t, z)$ and $e(t, z)$ to the only time dependent flow and effort approximations in the infinitesimal grid $f_{\mathscr{Z}_{a b c d}}(t), e_{\mathscr{Z}_{\text {abcd }}}(t)$ and the space weighting function $w_{\mathscr{Z}_{a b c d}}(z)$. For the sake of simplification, we omit $(t)$ and $(z)$ for the infinitesimal flows (efforts) variables and weighting functions respectively.

The flow variables are approximated as:

$$
\begin{gathered}
f^{\theta}(t, z)=f_{a b c d}^{\theta} w_{a b c d}^{\theta} \\
f^{\Gamma}(t, z)=f_{a b}^{\Gamma} w_{a b}^{\Gamma}+f_{b c}^{\Gamma} w_{b c}^{\Gamma}+f_{c d}^{\Gamma} w_{c d}^{\Gamma}+f_{d a}^{\Gamma} w_{d a}^{\Gamma}
\end{gathered}
$$


where the one-forms $w_{l}^{\Gamma}, l \in\{a b, b c, c d, d a\}$, and the two-form $w_{a b c d}^{\theta}$ satisfy the following conditions:

$$
\int_{l^{\prime}} w_{l}^{\Gamma}=\left\{\begin{array}{l}
0 \text { if } l^{\prime} \neq l, \\
1 \text { if } l^{\prime}=l,
\end{array} \int_{\mathscr{Z}_{a b c d}} w_{a b c d}^{\theta}=1\right.
$$

The effort variables, the velocity (zero-form) $e^{v}$ and the pressure (one form) $e^{p}$, are approximated as:

$$
\begin{gathered}
e^{v}(t, z)=e_{a}^{v} w_{a}^{v}+e_{b}^{v} w_{b}^{v}+e_{c}^{v} w_{c}^{v}+e_{d}^{v} w_{d}^{v} \\
e^{p}(t, z)=e_{a b}^{p} w_{a b}^{p}+e_{b c}^{p} w_{b c}^{p}+e_{c d}^{p} w_{c d}^{p}+e_{d a}^{p} w_{d a}^{p}
\end{gathered}
$$

where the one-forms $w_{l}^{p}, l \in\{a b, b c, c d, d a\}$, and the zero-form $w_{m}^{v}, m \in\{a, b, c, d\}$, satisfy the following conditions:

$$
\int_{l^{\prime}} w_{l}^{p}=\left\{\begin{array}{ll}
0 & \text { if } l^{\prime} \neq l, \\
1 & \text { if } l^{\prime}=l,
\end{array} \quad w_{m^{\prime}}^{v}(m)= \begin{cases}0 & m^{\prime} \neq m \\
1 & m^{\prime}=m\end{cases}\right.
$$

In order to write the dynamic equations of the 2-D vibro-acoustic system with the above finite dimensional Dirac structure, we have to define the weighting functions $w_{a b c d}, w_{l}$ with $l \in\{a b, b c, c d, d a\}$ and $w_{m}$ with $m \in\{a, b, c, d\}$ such that the parameters $\alpha, \beta$ and $\gamma$ of the Dirac structure are explicit. In order to get explicit system, in[Wu et al., 2015], we uses some simplified parameters $\alpha, \beta$ and $\gamma$ which do not satisfy the weighting function conditions (13), (16) and the compatibility conditions (68). In this paper, we will take all these conditions into account to achieve the explicit system.

Consider the infinitesimal square shown in Fig. 2. The position of every vertices is defined as $a:\left(x_{1}, y_{1}\right), b:\left(x_{2}, y_{1}\right), c:\left(x_{2}, y_{2}\right)$ and $d:\left(x_{1}, y_{2}\right)$. In this paper we consider as two forms weight

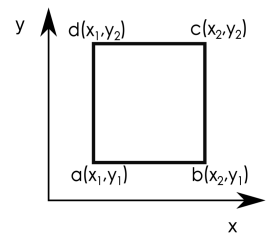

FIG. 2. An infinitesimal square grid

function:

$$
w_{a b c d}=\frac{1}{\left(x_{2}-x_{1}\right)\left(y_{2}-y_{1}\right)} d x \wedge d y
$$

which satisfies the equation (13). The one-form weighting functions $w_{l}$ with $l \in\{a b, b c, c d, d a\}$ and the zero-form weighting functions $w_{m}$ with $m \in\{a, b, c, d\}$ are defined as

$$
\begin{array}{cc}
w_{a b}=\frac{\left(y_{2}-y\right)}{\left(x_{2}-x_{1}\right)\left(y_{2}-y_{1}\right)} d x ; & w_{b c}=\frac{\left(x-x_{1}\right)}{\left(x_{2}-x_{1}\right)\left(y_{2}-y_{1}\right)} d y ; \\
w_{c d}=\frac{\left(y_{1}-y\right)}{\left(x_{2}-x_{1}\right)\left(y_{2}-y_{1}\right)} d x & w_{d a}=\frac{\left(x-x_{2}\right)}{\left(x_{2}-x_{1}\right)\left(y_{2}-y_{1}\right)} d y . \\
w_{a}=\frac{\left(x_{2}-x\right)}{\left(x_{2}-x_{1}\right)} \frac{\left(y_{2}-y\right)}{\left(y_{2}-y_{1}\right)} ; & w_{b}=\frac{\left(x-x_{1}\right)}{\left(x_{2}-x_{1}\right)} \frac{\left(y_{2}-y\right)}{\left(y_{2}-y_{1}\right)} ; \\
w_{c}=\frac{\left(x-x_{1}\right)}{\left(x_{2}-x_{1}\right)} \frac{\left(y-y_{1}\right)}{\left(y_{2}-y_{1}\right)} ; & w_{d}=\frac{\left(x_{2}-x\right)}{\left(x_{2}-x_{1}\right)} \frac{\left(y-y_{1}\right)}{\left(y_{2}-y_{1}\right)},
\end{array}
$$

and satisfy the equations (16). After some computation, one can check the compatibility conditions (68) are satisfied by using the above choice of weighting functions. 
With the above weighting functions, one can write the finite dimensional Dirac structure of an infinitesimal square grid (see computation details in the Appendix 1) as follow:

$$
\left[\begin{array}{cccccccc}
\frac{1}{4} & 0 & 0 & 0 & 0 & 0 & 0 & 0 \\
\frac{1}{4} & 0 & 0 & 0 & 0 & 0 & 0 & 0 \\
\frac{1}{4} & 0 & 0 & 0 & 0 & 0 & 0 & 0 \\
\frac{1}{4} & 0 & 0 & 0 & 0 & 0 & 0 & 0 \\
0 & -\frac{1}{4} & -\frac{1}{4} & -\frac{1}{2} & 1 & 0 & 0 & 0 \\
0 & -\frac{1}{4} & \frac{1}{4} & 0 & 0 & 1 & 0 & 0 \\
0 & \frac{1}{4} & \frac{1}{4} & \frac{1}{2} & 0 & 0 & 1 & 0 \\
0 & \frac{1}{4} & -\frac{1}{4} & 0 & 0 & 0 & 0 & 1
\end{array}\right]\left[\begin{array}{c}
f_{a b c d}^{\theta} \\
f_{a b}^{\Gamma} \\
f_{c d}^{I} \\
f_{d a}^{I} \\
f_{a b}^{B} \\
f_{b c}^{B} \\
f_{c d}^{B} \\
f_{d a}^{B}
\end{array}\right]+\left[\begin{array}{cccccccc}
0 & -1 & 0 & 1 & \frac{1}{2} & 0 & 0 & \frac{1}{2} \\
0 & 1 & 0 & 0 & \frac{1}{2} & \frac{1}{2} & 0 & 0 \\
0 & 0 & -1 & 0 & 0 & \frac{1}{2} & \frac{1}{2} & 0 \\
0 & 0 & 1 & -1 & 0 & 0 & \frac{1}{2} & \frac{1}{2} \\
-1 & 0 & 0 & 0 & 0 & 0 & 0 & 0 \\
-1 & 0 & 0 & 0 & 0 & 0 & 0 & 0 \\
-1 & 0 & 0 & 0 & 0 & 0 & 0 & 0 \\
-1 & 0 & 0 & 0 & 0 & 0 & 0 & 0
\end{array}\right]\left[\begin{array}{c}
e_{a b c d}^{\theta} \\
e_{a b}^{\Gamma} \\
e_{c d}^{I} \\
e_{d a}^{I} \\
e_{a b}^{B} \\
-e_{b c}^{B} \\
-e_{c d}^{B} \\
e_{d a}^{B}
\end{array}\right]=0 .
$$

\subsection{Discretization of $2 D$ vibro-acoustic system}

In the previous subsection, we discussed the approximation of the Dirac structure over an the infinitesimal square grid. In this section, we derive from this elementary model the explicit port-Hamiltonian formulation of the 2-D vibro-acoustic tube. We also include the dynamics of the membrane used for control and that is interconnected at boundary of the 2-D vibro-acoustic tube in the model. To this end, we consider the interconnection of each infinitesimal square (shown in Fig. 2) in two steps as shown in Fig. 3. First, the infinitesimal elements 0 to $N-1$ are vertically interconnected and then all the vertical elements $M$ are interconnected horizontally (like element $j$ to $j+1$ ) as shown in Fig. 3. We present the explicit input-output representation of each infinitesimal square and then the complete system with the vertical and horizontal interconnections.

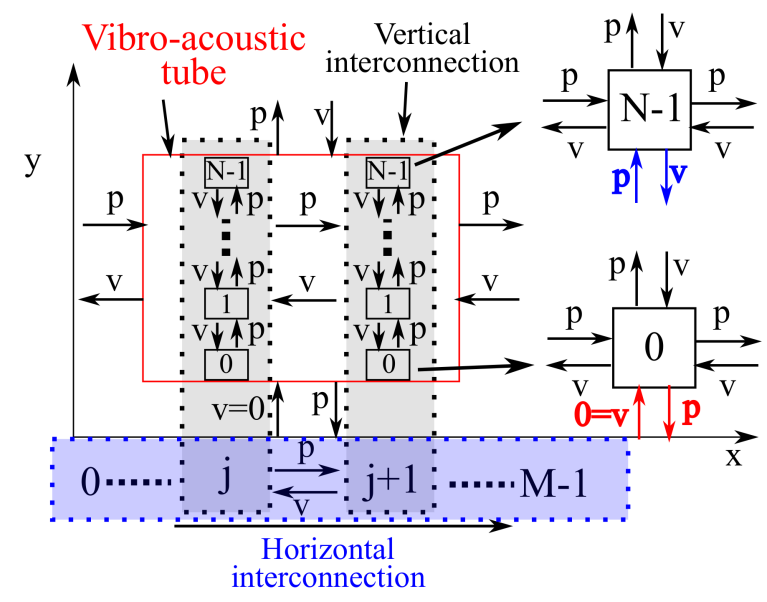

FIG. 3. Interconnection relation of vibro-acoustic tube

\subsection{Explicit input-output representation by interconnection}

The first step consists in interconnect the element 0 to $N-1$ vertically. However, we have to notice that the boundary conditions of the bottom element 0 is different from the ones of elements 1 to $N-1$. 
That is because the physical input of the vibro-acoustic tube is the pressure on the left side and the control variables on the top boundary of the tube is the velocity due to the control membrane interconnected to the tube. The output measurement of the tube is the pressure on the right side. From the above input-output consideration, the inputs of each element are the pressure on the left and bottom sides and the velocity on the right and top sides. However, from the axis symmetry assumption, the boundary condition on the bottom of the tube is the velocity $v=0$ which should be considered as the input. This boundary condition and the input-output consideration present a causality contradiction. In order to deal with this causality, a different input-output configuration shall be considered on element 0 .

With the above boundary conditions, we first consider the inputs and outputs of the explicit representation for the infinitesimal square grid of elements 1 to $N-1$ as follows:

$$
\left[\begin{array}{l}
u_{1} \\
u_{2} \\
u_{3} \\
u_{4}
\end{array}\right]=\left[\begin{array}{c}
e_{a b}^{B} \\
f_{b c}^{B} \\
f_{c d}^{B} \\
e_{d a}^{B}
\end{array}\right]=\left[\begin{array}{c}
p_{a b} \\
v_{b c} \\
v_{c d} \\
p_{d a}
\end{array}\right], \quad\left[\begin{array}{c}
y_{1} \\
y_{2} \\
y_{3} \\
y_{4}
\end{array}\right]=\left[\begin{array}{c}
f_{a b}^{B} \\
-e_{b c}^{B} \\
-e_{c d}^{B} \\
f_{d a}^{B}
\end{array}\right]=\left[\begin{array}{c}
v_{a b} \\
p_{b c} \\
p_{c d} \\
v_{d a}
\end{array}\right] .
$$

Taking into account the above input-output variables and the Dirac structure of the infinitesimal square (20) with the following dynamics:

$$
\left[\begin{array}{l}
-\dot{x}_{1} \\
-\dot{x}_{2} \\
-\dot{x}_{3} \\
-\dot{x}_{4}
\end{array}\right]=\left[\begin{array}{l}
f_{1} \\
f_{2} \\
f_{3} \\
f_{4}
\end{array}\right]=\left[\begin{array}{l}
f_{a b c d}^{\theta} \\
f_{a b}^{I} \\
f_{c d}^{I} \\
f_{d a}^{T}
\end{array}\right],\left[\begin{array}{c}
e_{1} \\
e_{2} \\
e_{3} \\
e_{4}
\end{array}\right]=\left[\begin{array}{c}
e_{a b c d}^{\theta} \\
e_{a b}^{\Gamma} \\
e_{c d}^{I} \\
e_{d a}^{I}
\end{array}\right],
$$

the explicit port-Hamiltonian representation for the elements 1 to $N-1$ can be written as:

$$
\left\{\begin{array}{l}
{\left[\begin{array}{l}
\dot{x}_{1} \\
\dot{x}_{2} \\
\dot{x}_{4}
\end{array}\right]=\left[\begin{array}{ccc}
0 & -4 & 4 \\
4 & 0 & 0 \\
-4 & 0 & 0
\end{array}\right]\left[\begin{array}{l}
e_{1} \\
e_{2} \\
e_{4}
\end{array}\right]+\left[\begin{array}{cccc}
2 & 0 & 0 & 2 \\
0 & -4 & 0 & 0 \\
0 & 2 & 2 & 0
\end{array}\right]\left[\begin{array}{l}
u_{1} \\
u_{2} \\
u_{3} \\
u_{4}
\end{array}\right]} \\
{\left[\begin{array}{l}
y_{1} \\
y_{2} \\
y_{3} \\
y_{4}
\end{array}\right]=\left[\begin{array}{ccc}
2 & 0 & 0 \\
0 & -4 & 2 \\
0 & 0 & 2 \\
2 & 0 & 0
\end{array}\right]\left[\begin{array}{l}
e_{1} \\
e_{2} \\
e_{4}
\end{array}\right]+\left[\begin{array}{cccc}
0 & 0 & -1 & 0 \\
0 & 0 & 0 & 1 \\
1 & 0 & 0 & 0 \\
0 & -1 & 0 & 0
\end{array}\right]\left[\begin{array}{l}
u_{1} \\
u_{2} \\
u_{3} \\
u_{4}
\end{array}\right]}
\end{array}\right.
$$

One can notice that the above system does not have dynamics in $\dot{x}_{3}$. This is due to the explicit formulation the Dirac structure (20). The constitutive relations (See the details of the computation in the Appendix 2) are:

$$
\left[\begin{array}{l}
e_{1} \\
e_{2} \\
e_{4}
\end{array}\right]=\underbrace{\left[\begin{array}{ccc}
S_{\text {in }} \frac{1}{\rho_{0}} & 0 & 0 \\
0 & \frac{1}{3} S_{2 i n} \frac{1}{\chi_{s}} & -S_{1 i n} \frac{1}{6 \chi_{s}} \\
0 & -S_{\operatorname{lin}} \frac{1}{6 \chi_{s}} & S_{\operatorname{lin}} \frac{1}{\chi_{s}}
\end{array}\right]}_{Q}\left[\begin{array}{l}
x_{1} \\
x_{2} \\
x_{4}
\end{array}\right]
$$

The Hamiltonian function of each element is defined as $H=\frac{1}{2} x^{T} Q x$. For the purpose of simplicity, we take the notation $\left[\begin{array}{lll}x_{1} & x_{2} & x_{3}\end{array}\right]^{T}$ and $\left[\begin{array}{lll}e_{1} & e_{2} & e_{3}\end{array}\right]^{T}$ instead of $\left[\begin{array}{lll}x_{1} & x_{2} & x_{4}\end{array}\right]^{T}$ and $\left[\begin{array}{lll}e_{1} & e_{2} & e_{4}\end{array}\right]^{T}$. 
Now we consider the explicit representation of the bottom system 0 from the kernel representation of the Dirac structure (20) with the following choice of inputs and outputs:

$$
\left[\begin{array}{l}
u_{1} \\
u_{2} \\
u_{3} \\
u_{4}
\end{array}\right]=\left[\begin{array}{l}
f_{a b}^{B} \\
f_{b c}^{B} \\
f_{c d}^{B} \\
e_{d a}^{B}
\end{array}\right]=\left[\begin{array}{l}
v_{a b} \\
v_{b c} \\
v_{c d} \\
p_{d a}
\end{array}\right], \quad\left[\begin{array}{l}
y_{1} \\
y_{2} \\
y_{3} \\
y_{4}
\end{array}\right]=\left[\begin{array}{c}
e_{a b}^{B} \\
-e_{b c}^{B} \\
-e_{c d}^{B} \\
f_{d a}^{B}
\end{array}\right]=\left[\begin{array}{c}
p_{a b} \\
p_{b c} \\
p_{c d} \\
v_{d a}
\end{array}\right]
$$

One can notice that the difference in inputs and outputs between the bottom element and the other elements are $u_{1}$ and $y_{1}$. In the same manner, the explicit formulation for the element 0 is given by:

$$
\left\{\begin{array}{l}
{\left[\begin{array}{l}
\dot{x}_{1} \\
\dot{x}_{2} \\
\dot{x}_{3}
\end{array}\right]=\left[\begin{array}{ccc}
0 & -4 & 4 \\
4 & 0 & 0 \\
-4 & 0 & 0
\end{array}\right]\left[\begin{array}{l}
e_{1} \\
e_{2} \\
e_{3}
\end{array}\right]+\left[\begin{array}{cccc}
0 & 0 & 0 & 2 \\
0 & -4 & 0 & 0 \\
2 & 2 & 2 & 0
\end{array}\right]\left[\begin{array}{l}
u_{1} \\
u_{2} \\
u_{3} \\
u_{4}
\end{array}\right]} \\
{\left[\begin{array}{l}
y_{1} \\
y_{2} \\
y_{3} \\
y_{4}
\end{array}\right]=\left[\begin{array}{ccc}
1 & 0 & 0 \\
0 & -4 & 2 \\
0 & 0 & 2 \\
0 & 0 & 0
\end{array}\right]\left[\begin{array}{l}
e_{1} \\
e_{2} \\
e_{3}
\end{array}\right]+\left[\begin{array}{cccc}
0 & 0 & 0 & 0 \\
0 & 0 & 0 & 1 \\
0 & 0 & 0 & 0 \\
0 & -1 & 0 & 0
\end{array}\right]\left[\begin{array}{l}
u_{1} \\
u_{2} \\
u_{3} \\
u_{4}
\end{array}\right]}
\end{array}\right.
$$

In order to get vertical interconnection of all elements, the following interconnection relation is considered:

$$
\begin{aligned}
& u_{3}^{i}=y_{1}^{i+1} \\
& u_{1}^{i+1}=-y_{3}^{i}
\end{aligned} \quad \text { with } \quad i=0, \ldots, N-2
$$

Thus the vertically interconnected explicit system:

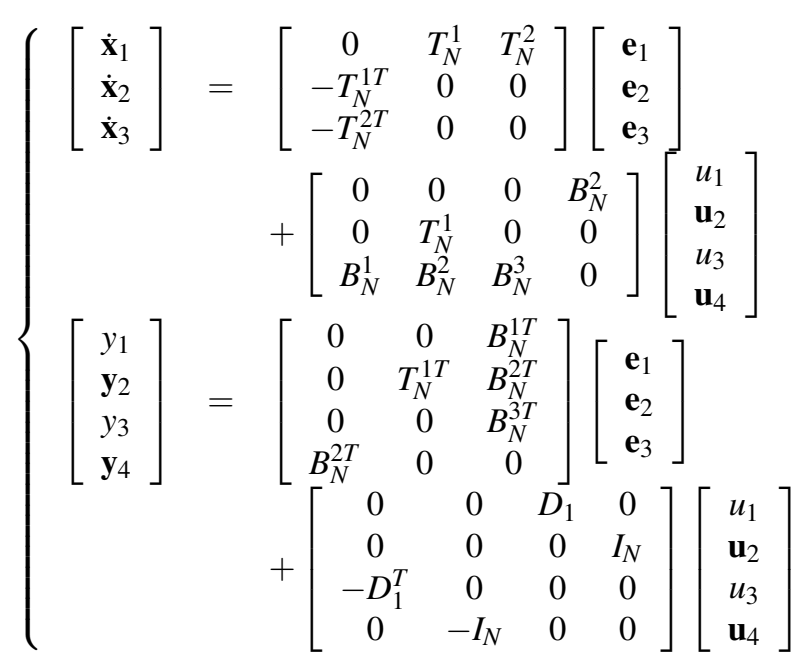

where 0 represents zero matrices of appropriate dimension and the state variables are $\mathbf{x}_{i}=\left[x_{i}^{1}, x_{i}^{2}, \ldots, x_{i}^{N-1}\right]^{T}$ with $i \in\{1,2,3\}$. The matrices are defined as

$$
T_{N}^{1}=\operatorname{diag}[4,4, \cdots, 4] \in \mathbb{R}^{N \times N},
$$




$$
\begin{gathered}
T_{N}^{2}=\left[\begin{array}{ccccc}
-4 & 0 & 0 & \cdots & 0 \\
4 & -4 & 0 & \ddots & \vdots \\
-4 & 4 & -4 & \ddots & 0 \\
\vdots & \ddots & \ddots & \ddots & 0 \\
(-1)^{N} 4 & \cdots & -4 & 4 & -4
\end{array}\right] \in \mathbb{R}^{N \times N} \\
B_{N}^{1}=\left[\begin{array}{c}
-2 \\
2 \\
\vdots \\
(-1)^{N} 2
\end{array}\right], B_{N}^{2}=\operatorname{diag}[-2,-2, \cdots,-2] \in \mathbb{R}^{N \times N}, \\
B_{N}^{3}=\left[\begin{array}{c}
(-1)^{N-1} 2 \\
\vdots \\
-2 \\
2
\end{array}\right], D_{1}=\left[\begin{array}{c}
-1 \\
0 \\
\vdots \\
0
\end{array}\right] \in \mathbb{R}^{N} .
\end{gathered}
$$

The inputs and outputs of each vertically interconnected explicit system are defined as follows: the pressure inputs and velocity outputs at the left side and the top of the tube, the velocity inputs and the pressure measurement at the right side and the bottom of the tube,

$$
\begin{gathered}
u_{1}=u_{1}^{0}=v_{a b}^{0} ; y_{1}=y_{1}^{0}=p_{a b}^{0} ; \\
\mathbf{u}_{2}=\left[\begin{array}{c}
v_{b c}^{0} \\
v_{b c}^{1} \\
\vdots \\
v_{b c}^{N-1}
\end{array}\right] ; \mathbf{y}_{2}=\left[\begin{array}{c}
p_{b c}^{0} \\
p_{b c}^{1} \\
\vdots \\
p_{b c}^{N-1}
\end{array}\right] ; \\
u_{3}=u_{3}^{N-1}=v_{c d}^{N-1} ; y_{3}=y_{3}^{N-1}=p_{c d}^{N-1} ;
\end{gathered}
$$

The left side of each vertically interconnected system has the pressure as input which corresponds to the physical input and its power conjugate output is the corresponding velocity:

$$
\mathbf{u}_{4}=\left[\begin{array}{c}
p_{d a}^{0} \\
p_{d a}^{1} \\
\vdots \\
p_{d a}^{N-1}
\end{array}\right] ; \mathbf{y}_{4}=\left[\begin{array}{c}
v_{d a}^{0} \\
v_{d a}^{1} \\
\vdots \\
v_{d a}^{N-1}
\end{array}\right]
$$

Now we discuss the horizontal interconnection of each obtained vertical element (28). The input on the left side of each vertical element is the pressure while it is the output on the right side. Thus, the interconnection relation between each vertical element (28) is given as:

$$
\begin{aligned}
& \mathbf{u}_{2}^{j}=\mathbf{y}_{4}^{j+1} \\
& \mathbf{u}_{4}^{j+1}=-\mathbf{y}_{2}^{j}
\end{aligned} \quad \text { with } \quad j=0, \ldots, M-2 .
$$


10 of 22

With the above relations, we obtain the interconnected system:

$$
\left\{\begin{array}{l}
{\left[\begin{array}{c}
\dot{\tilde{\mathbf{x}}}_{1} \\
\tilde{\tilde{\mathbf{x}}}_{2} \\
\tilde{\mathbf{x}}_{3}
\end{array}\right]=\mathbf{J}\left[\begin{array}{c}
\tilde{\mathbf{e}}_{1} \\
\tilde{\mathbf{e}}_{2} \\
\tilde{\mathbf{e}}_{3}
\end{array}\right]+\mathbf{B}\left[\begin{array}{l}
\mathbf{u}_{1} \\
\mathbf{u}_{2} \\
\mathbf{u}_{3} \\
\mathbf{u}_{4}
\end{array}\right]} \\
{\left[\begin{array}{l}
\mathbf{y}_{1} \\
\mathbf{y}_{2} \\
\mathbf{y}_{3} \\
\mathbf{y}_{4}
\end{array}\right]=\mathbf{B}^{T}\left[\begin{array}{c}
\tilde{\mathbf{e}}_{1} \\
\tilde{\mathbf{e}}_{2} \\
\tilde{\mathbf{e}}_{3}
\end{array}\right]+\mathbf{D}\left[\begin{array}{l}
\mathbf{u}_{1} \\
\mathbf{u}_{2} \\
\mathbf{u}_{3} \\
\mathbf{u}_{4}
\end{array}\right]}
\end{array}\right.
$$

where the state variable are $\tilde{\mathbf{x}}_{i}=\left[\mathbf{x}_{i}^{0}, \mathbf{x}^{1}, \ldots, \mathbf{x}_{i}^{M-1}\right]^{T}$ with $i \in\{1,2,3\}$. The constitutive relation of the discretized system can be written as:

$$
\tilde{\mathbf{e}}=\left[\begin{array}{ccc}
S_{1} & 0 & 0 \\
0 & S_{2} & S_{4} \\
0 & S_{4}^{T} & S_{3}
\end{array}\right] \mathbf{x}=\tilde{\mathbf{Q}} \mathbf{x}
$$

with $S_{1}=\operatorname{diag}\left[S_{i n} \frac{1}{\rho_{0}}\right], S_{2}=\operatorname{diag}\left[\frac{1}{3} S_{2 i n} \frac{1}{\chi_{s}}\right], S_{3}=\operatorname{diag}\left[S_{1 i n} \frac{1}{\chi_{s}}\right]$, and $S_{4}=\operatorname{diag}\left[-S_{1 i n} \frac{1}{6 \chi_{s}}\right]$.

The inputs are the velocity at the bottom $\{a b\}$, the right side $\{b c\}$ and the top $\{c d\}$ of the tube, respectively, $\mathbf{u}_{1}=\left[v_{a b}^{0}, v_{a b}^{1}, \ldots, v_{a b}^{M-1}\right]^{T}, \mathbf{u}_{2}=\left[v_{b c}^{0}, v_{b c}^{1}, \ldots, v_{b c}^{N-1}\right]^{T}, \mathbf{u}_{3}=\left[v_{c d}^{0}, v_{c d}^{1}, \ldots, v_{c d}^{M-1}\right]^{T}$, and the pressure on the right side $\{d a\}$ of the tube, $\mathbf{u}_{4}=\left[p_{d a}^{0}, p_{d a}^{1}, \ldots, p_{d a}^{N-1}\right]^{T}$. The outputs are the power conjugate variables of the inputs, i.e., the pressure on the top, the bottom and the right side, the velocity on the left side of the vibro-acoustic tube. The interconnection matrix, input matrix and the feedforward matrix are given by:

$$
\begin{gathered}
\mathbf{J}=\left[\begin{array}{ccc}
0 & \tilde{T}_{1 M} & \tilde{T}_{2 M} \\
-\tilde{T}_{1 M}^{T} & 0 & 0 \\
-\tilde{T}_{2 M}^{T} & 0 & 0
\end{array}\right] ; \\
\mathbf{B}=\left[\begin{array}{cccc}
0 & 0 & 0 & -\bar{B}_{M}^{2} \\
0 & T_{M}^{1 B} & 0 & 0 \\
\tilde{B}_{M}^{1} & \tilde{B}_{M}^{2} & \tilde{B}_{M}^{3} & 0
\end{array}\right] ; \\
\mathbf{D}=\left[\begin{array}{cccc}
0 & 0 & 0 & 0 \\
0 & 0 & 0 & (-1)^{M-1} I_{N \times N} \\
0 & 0 & 0 & 0 \\
0 & (-1)^{M} I_{N \times N} & 0 & 0
\end{array}\right]
\end{gathered}
$$

with 0 zero matrices of appropriate dimension and the sub-matrices are given by:

$$
\tilde{T}_{1 M}=\left[\begin{array}{cccc}
T_{1 N} & 0 & \cdots & 0 \\
-B_{N}^{2} T_{1 N}^{T} & T_{1 N} & \ddots & \vdots \\
\vdots & \ddots & \ddots & 0 \\
(-1)^{M-1} B_{N}^{2} T_{1 N}^{T} & \cdots & -B_{N}^{2} T_{1 N}^{T} & T_{1 N}
\end{array}\right] ;
$$




$$
\begin{gathered}
\tilde{T}_{2 M}=\left[\begin{array}{cccc}
T_{2 N} & 0 & \cdots & 0 \\
-B_{N}^{2} B_{N}^{2 T} & T_{2 N} & \ddots & \vdots \\
\vdots & \ddots & \ddots & 0 \\
(-1)^{M-1} B_{N}^{2} B_{N}^{2 T} & \cdots & -B_{N}^{2} B_{N}^{2 T} & T_{2 N}
\end{array}\right] ; \\
T_{M}^{1 B}=\left[\begin{array}{c}
(-1)^{M-1} T_{N}^{1} \\
\vdots \\
-T_{N}^{1} \\
T_{N}^{1}
\end{array}\right] ; \bar{B}_{M}^{1}=\operatorname{diag}\left[B_{N}^{1}\right] ; \\
\tilde{B}_{M}^{2}=\left[\begin{array}{c}
(-1)^{M-1} B_{N}^{2} \\
\vdots \\
-B_{N}^{2} \\
B_{N}^{2}
\end{array}\right] ; \tilde{B}_{M}^{3}=\operatorname{diag}\left[B_{N}^{3}\right] .
\end{gathered}
$$

\section{Control by interconnection}

In this section, we will consider the control design for the discretised model (38) by using the energy shaping based on Casimir invariants. we consider that the vibro-acoustic tube can be actuated allover its length by a control surface, as shown in Fig. 4. From the axis-symmetry assumption, the control surface actuates only on the top $\{b c\}$ of the 2-D vibro-acoustic system.

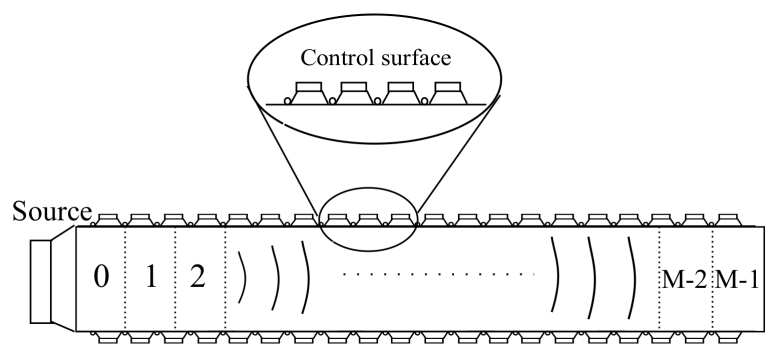

FIG. 4. Control of vibro-acoustic tube via Interconnection

The control surface is composed by a set of micro microphone-loudspeakers which are used to actively attenuate the wave pressure in the tube or to change the wave properties in a desired manner. The control is effectuated by the flexible membrane of the micro-loudspeakers which are distributed along the tube. We consider that the flexible membrane is a simple mass spring system interconnected with the vibro-acoustic tube. The inputs of the membrane are the external control force and the acoustic wave pressure. The system can be written in the following form:

$$
\left\{\begin{aligned}
& {\left[\begin{array}{l}
\dot{x}_{m} \\
\dot{p}_{m}
\end{array}\right] }=\left[\begin{array}{cc}
0 & I \\
-I & 0
\end{array}\right]\left[\begin{array}{l}
\frac{\partial H_{m}}{\partial x_{m}} \\
\frac{\partial H_{m}}{\partial m_{m}}
\end{array}\right]+\left[\begin{array}{ll}
0 & 0 \\
I & I
\end{array}\right]\left[\begin{array}{l}
F \\
P_{s}
\end{array}\right] \\
& {\left[\begin{array}{l}
y_{F} \\
y_{s}
\end{array}\right]=\left[\begin{array}{ll}
0 & I \\
0 & I
\end{array}\right]\left[\begin{array}{l}
\frac{\partial H_{m}}{\partial x_{m}} \\
\frac{\partial H_{m}}{\partial p_{m}}
\end{array}\right] }
\end{aligned}\right.
$$


with Hamiltonian functional $H_{m}\left(x_{m}, p_{m}\right)=\frac{1}{2} k_{m} x_{m}^{2}+\frac{1}{2} \frac{1}{m} p_{m}^{2}$ where $k_{m}$ and $m$ are the stiffness and mass of the membrane respectively. We consider each vertical element of the system (38) interconnected with a mass-spring-damper system. Thus, the state variables $\left[\begin{array}{ll}x_{m} & p_{m}\end{array}\right]^{T} \in \mathbb{R}^{2 M} . F \mathbb{R}^{M}$ are the control forces and $P_{s} \mathbb{R}^{M}$ are the forces generated by the wave pressure on the micro-loudspeaker. The $y_{F} \in \mathbb{R}^{M}$ and $y_{s} \in \mathbb{R}^{M}$ are the power conjugate velocities. The interconnection relation of the 2-D vibro-acoustic wave system (38) and the membrane model (43) is :

$$
\begin{aligned}
\mathbf{u}_{3} & =-y_{s} \\
P_{s} & =\mathbf{y}_{3}
\end{aligned}
$$

The interconnected system can be written as:

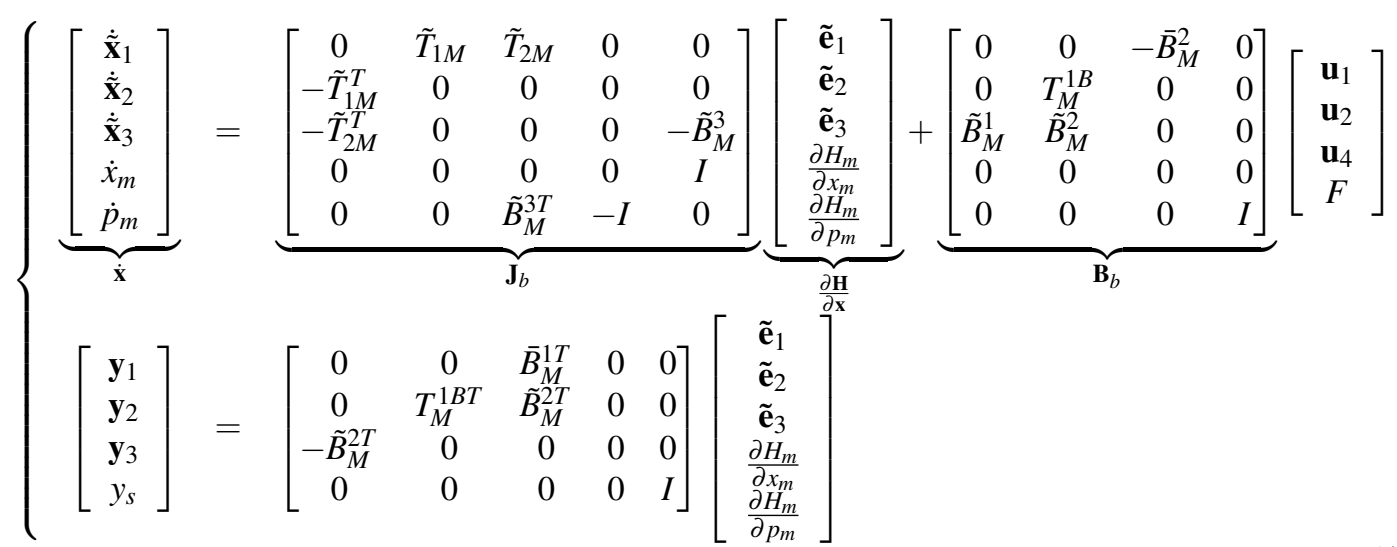

where the Hamiltonian of the system is defined as $\mathbf{H}\left(\tilde{\mathbf{x}}, x_{m}, p_{m}\right)=\frac{1}{2} \tilde{\mathbf{x}}^{T} \tilde{\mathbf{Q}} \tilde{\mathbf{x}}+\frac{1}{2} k_{m} x_{m}^{2}+\frac{1}{2} \frac{1}{m} p_{m}^{2}=\frac{1}{2} \mathbf{x}^{T} \mathbf{Q} \mathbf{x}$.

Now we want to derive a control law which attenuates the wave pressure controlling the membrane of the micro-loudspeaker. To this end we shall employ the well known control by interconnection approach, in which the existence of closed-loop invariants are used to synthesis a controller which rendes the closed-loop Hamiltonian function into a desired form. A detailed survey on this approach can be found in [van der Schaft, 2000]. Consider the following port Hamiltonian controller:

$$
\left\{\begin{aligned}
\dot{\xi} & =\left(J_{c}(\xi)-R(\xi)\right) \frac{\partial H_{c}}{\partial \xi}(\xi)+g_{c}(\xi) u_{c} \\
y_{c} & =g_{c}^{T}(\xi) \frac{\partial H_{c}}{\partial \xi}(\xi)
\end{aligned}\right.
$$

with state variable $\xi \in \mathbb{R}^{M}$, Hamiltonian function $H_{c}(\xi)$ and the structure matrices $J_{c}(\xi)=-J_{c}(\xi)^{T}$ and $R_{c}(\xi)=R_{c}(\xi)^{T} \geqslant 0$. The controller and the system are interconnected at the top of the tube with the membrane. Hence the power preserving interconnection of the controller and the system is:

$$
\begin{aligned}
F & =-y_{c} \\
u_{c} & =y_{s}
\end{aligned}
$$

Thus, the coupling system is still passive and can be written as:

$$
\left[\begin{array}{l}
\dot{\mathbf{x}} \\
\dot{\xi}
\end{array}\right]=\left(\left[\begin{array}{cc}
\mathbf{J}_{\mathbf{b}} & -g_{c}^{T}(\xi) \\
g_{c}(\xi) & J_{c}(\xi)
\end{array}\right]-\left[\begin{array}{cc}
0 & 0 \\
0 & R_{c}(\zeta)
\end{array}\right]\right)\left[\begin{array}{l}
\frac{\partial H_{d}}{\partial \mathbf{x}}(\mathbf{x}) \\
\frac{\partial H_{d}}{\partial \xi}(\xi)
\end{array}\right]
$$


where $H_{d}(\tilde{\mathbf{x}}, \xi)=\mathbf{H}(\tilde{\mathbf{x}})+H_{c}(\xi)$ is the energy of the closed-loop system.

In order to shape the closed-loop energy, we need to relate the state of the controller $\xi$ to the state of the system $\mathbf{x}$. We define the Casimir functions as follows

$$
C(\mathbf{x}, \xi)=F(\mathbf{x})-\xi .
$$

These Casimirs functions are invariant quantities along the closed-loop system trajectories independent from the energy function $H_{d}$, i.e.,

$$
\dot{C}(\mathbf{x}, \xi)=\left[\begin{array}{ll}
\frac{\partial^{T} F}{\partial \mathbf{x}} & -I
\end{array}\right]\left[\begin{array}{cc}
\mathbf{J}_{\mathbf{b}} & g_{c}^{T} \\
g_{c} & J_{c}-R_{c}
\end{array}\right]\left[\begin{array}{c}
\frac{\partial H_{d}}{\partial \mathbf{x}}(\mathbf{x}) \\
\frac{\partial H_{d}}{\partial \xi}(\xi)
\end{array}\right]=0
$$

which implies the following matching equations:

$$
\begin{array}{r}
\frac{\partial^{T} F}{\partial \mathbf{x}}(\mathbf{x}) \mathbf{J}_{\mathbf{b}} \frac{\partial F}{\partial \mathbf{x}}(\mathbf{x})=J_{c}(\xi) \\
R_{c}=0 \\
\frac{\partial^{T} F}{\partial \mathbf{x}}(\mathbf{x}) \mathbf{J}_{\mathbf{b}}=g_{c}
\end{array}
$$

Notice that the system (38) does not have dissipation, hence the dissipation obstacle [Ortega et al., 2001] is avoided. Developing the matching equations (51) we obtain:

$$
\begin{array}{r}
\frac{\partial^{T} F}{\partial \mathbf{x}_{1}} \tilde{T}_{1 M}=0 \\
\frac{\partial^{T} F}{\partial \mathbf{x}_{1}} \tilde{T}_{2 M}+\frac{\partial^{T} F}{\partial p_{m}} \tilde{B}_{M}^{3 T}=0 \\
-\frac{\partial^{T} F}{\partial p_{m}}=0 \\
\frac{\partial^{T} F}{\partial \mathbf{x}_{2}} \tilde{T}_{1 M}^{T}+\frac{\partial^{T} F}{\partial \mathbf{x}_{3}} \tilde{T}_{2 M}^{T}=0 \\
\frac{\partial^{T} F}{\partial \mathbf{x}_{3}} \tilde{B}_{M}^{3}+\frac{\partial^{T} F}{\partial x_{m}}=-g_{c}
\end{array}
$$

From the equations (53)-(55), we can tell that the function $F(\mathbf{x})$ does not depend on the variables $\mathbf{x}_{1}$ and $p_{m}$. Taking $g_{c}=I$ and the matrices defined in (38), a possible solution of the matching condition is:

$$
F(\mathbf{x})=-\frac{1}{2} \tilde{B}_{M}^{3 \dagger} \tilde{T}_{2 M}^{T} \tilde{T}_{1 M}^{-T} \mathbf{x}_{2}+\frac{1}{2} \tilde{B}_{M}^{3 \dagger} \mathbf{x}_{3}+\frac{1}{2} x_{m}
$$

where $\tilde{B}_{M}^{3 \dagger}$ is the pseudo inverse of $\tilde{B}_{M}^{3}$. A simple choice for the Casimir function is $C(\mathbf{x}, \xi)=0$ which implies the the following relation between system and controller states:

$$
\xi=F(\mathbf{x})=-\frac{1}{2} \tilde{B}_{M}^{3 \dagger} \tilde{T}_{2 M}^{T} \tilde{T}_{1 M}^{-T} \mathbf{x}_{2}+\frac{1}{2} \tilde{B}_{M}^{3 \dagger} \mathbf{x}_{3}+\frac{1}{2} x_{m}
$$

The state of the controller is related to $\mathbf{x}_{2}, \mathbf{x}_{3}$ and $x_{m}$, hence the energy can be shaped in these coordinates. To this end define the Hamiltonian of the controller as

$$
H_{c}(\xi)=2 K \xi^{2}
$$


This Hamiltonian allows to shape the energy $H_{d}$ around the zero equilibrium position with $K>0$ a control design parameter. Following the matching equations (50) and (51), the matrices $J_{c}=R_{c}=0$ and $g_{c}=I$. Then the controller is:

$$
\begin{array}{r}
\dot{\xi}=u_{c} \\
y_{c}=\frac{\partial H_{c}}{\partial \xi}
\end{array}
$$

Considering (59), the Hamiltonian of the controller can be written as :

$$
\begin{aligned}
H_{c}\left(\mathbf{x}_{2}, \mathbf{x}_{2}, x_{m}\right)= & \frac{1}{2} \mathbf{x}_{2}^{T} S_{c 2} \mathbf{x}_{2}+\frac{1}{2} \mathbf{x}_{3}^{T} S_{c 3} \mathbf{x}_{3}+\frac{1}{2} x_{m}^{T} k_{c} x_{m} \\
& +\mathbf{x}_{2}^{T} S_{c 4} \mathbf{x}_{3}+\mathbf{x}_{3}^{T} S_{c 5} x_{4}+\mathbf{x}_{2}^{T} S_{c 6} x_{4}
\end{aligned}
$$

where $S_{c 2}=\tilde{T}_{1 M}^{-1} \tilde{T}_{2 M} \tilde{B}_{M}^{3 \dagger T} K \tilde{B}_{M}^{3 \dagger} \tilde{T}_{2 M}^{T} \tilde{T}_{1 M}^{-T}, S_{c 3}=\tilde{B}_{M}^{3 \dagger T} K \tilde{B}_{M}^{3 \dagger}, k_{c}=K, S_{c 4}=\tilde{T}_{1 M}^{-1} \tilde{T}_{2 M} \tilde{B}_{M}^{3 \dagger T} K \tilde{B}_{M}^{3 \dagger}, S_{c 5}=\tilde{T}_{1 M}^{-1} \tilde{T}_{2 M} \tilde{B}_{M}^{3 \dagger T} K$ and $S_{c 6}=\tilde{B}_{M}^{3 \dagger T} K$.

From (61), (59) and (47), the control law can be written as:

$$
u=-y_{c}=2 K \tilde{B}_{M}^{3 \dagger} \tilde{T}_{2 M}^{T} \tilde{T}_{1 M}^{-T} \mathbf{x}_{2}-2 K \tilde{B}_{M}^{3 \dagger} \mathbf{x}_{3}-2 K x_{m}
$$

with closed loop Hamiltonian

$$
\begin{aligned}
H_{d}= & \frac{1}{2} \mathbf{x}_{1}^{T} S_{1} \mathbf{x}_{1}+\frac{1}{2} \mathbf{x}_{2}^{T} \bar{S}_{2} \mathbf{x}_{2}+\frac{1}{2} \mathbf{x}_{3}^{T} \bar{S}_{3} \mathbf{x}_{3}+\frac{1}{2} x_{m}^{T} \bar{k} x_{m}+\frac{1}{2} p_{m}^{T} \frac{1}{m} p_{m} \\
& +\mathbf{x}_{2}^{T} S_{c 4} \mathbf{x}_{3}+\mathbf{x}_{3}^{T} S_{c 5} x_{4}+\mathbf{x}_{2}^{T} S_{c 6} x_{4}
\end{aligned}
$$

with $\bar{S}_{2}=S_{2}+S_{c 2}, \bar{S}_{3}=S_{3}+S_{c 3}$ and $\bar{k}=K+k$. Furthermore, by adding a negative output feedback to the boundary feedback we introduce dissipation into the closed-loop system and guarantee the closedloop asymptotic stability [Macchelli et al., 2017]:

$$
u^{*}=u-\alpha y_{s}
$$

with the dissipation feedback coefficient $\alpha>0$.

\section{Numerical simulations}

In order to illustrate the numerical effectiveness of the proposed discretization scheme and the simple control strategy, we simulate the $2 \mathrm{D}$ wave propagation in the vibro-acoustic tube with the numerical parameters given in Table 1 [David et al., 2010].

\begin{tabular}{ccc} 
& Table 1. The parameters of the experimental tube \\
\hline \hline$L$ & Length & $1.84 \mathrm{~m}$ \\
$R$ & Radius & $0.05 \mathrm{~m}$ \\
$\rho$ & Air density & $0.8163 \mathrm{~kg} / \mathrm{m}^{3}$ \\
$\chi_{s}$ & Compressibility coefficient & $1.4161 \times 10^{5} \mathrm{~Pa}^{-1}$ \\
\hline \hline
\end{tabular}

For applying the previously proposed discretization scheme, we take 5 vertical elements i.e., $N=5$ and 250 horizontal elements i.e., $M=250$. Hence, the overall system has $M \times N=1250$ infinitesimal elements and 3750 state variables $\tilde{\mathbf{x}} \in \mathbb{R}^{3750}$. In Fig. 5, we show the open loop response of the pressure on the complete spatial domain at 5 second. The input is the wave pressure on the left side of the tube generated by the loudspeaker in sinusoidal form $u=\sin (100 * t)$. 


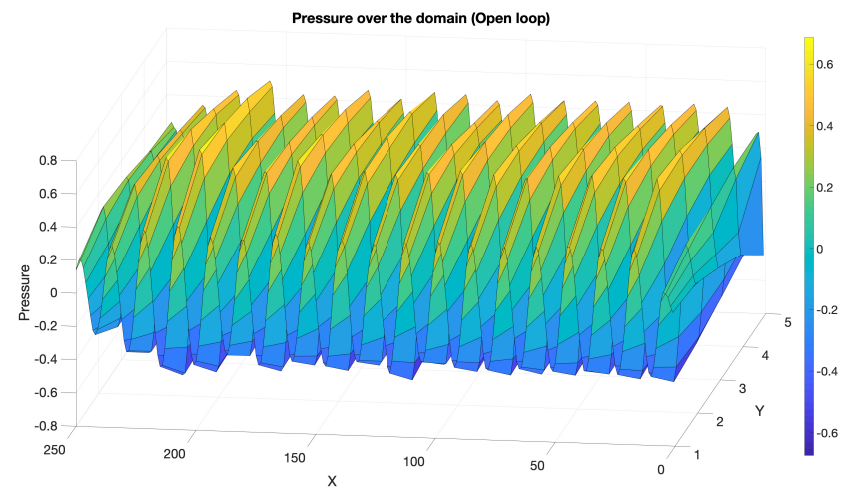

FIG. 5. Open loop pressure response over the $2 \mathrm{D}$ domain at $5 \mathrm{~s}$

In Fig. 6, the closed-loop response with the passive controller proposed in the last section is shown. To illustrate the effectiveness of the proposed control, we consider the the front of the tube (first 100th elements) is not controlled and the controller is star from the the element 100 to the end. The pressure over the spatial domain at 5 second is shown in Fig. 6. We observe that until the 100th element, the wave is not reduced. However, starting from element 101 the pressure is attenuated significantly because of the controller.

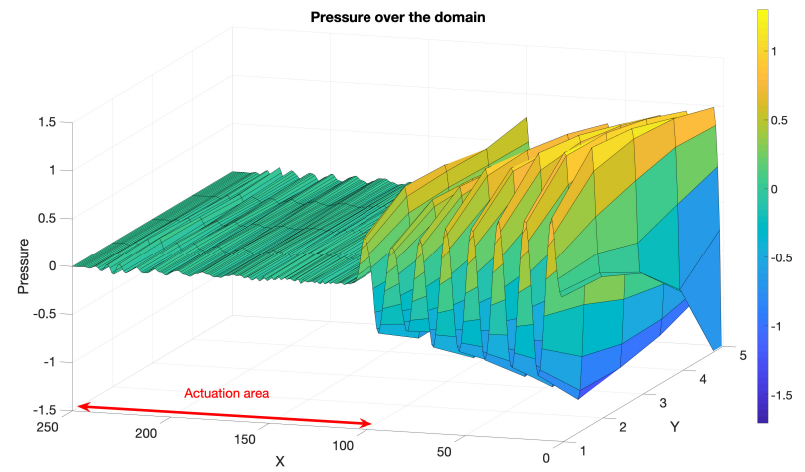

FIG. 6. Closed-loop pressure response over the 2D domain at $0.5 \mathrm{~s}$

\section{Conclusion}

A geometric structure preserving discretization for a $2 \mathrm{D}$ vibro-acoustic system under the port Hamiltonian form is addressed in this paper. With a precise choice of a set of weighting functions, a finite- 
dimensional Dirac structure is derived using a mixed finite-elements method and an explicit input-output system representation of the vibro-acoustic tube is achieved. The advantage is that, in the finite dimensional approximation of the vibro-acoustic system, the passivity and the Hamiltonian structure is preserved which is useful for control design. Furthermore, a passive controller via interconnection energy shaping and damping injection is implemented on the obtained finite dimensional approximation. The numerical result shows the effectiveness of the proposed discretization scheme and the passive controller. Ongoing work is on the implementation of the proposed simple controller on real experimental set-up

\section{References}

A. Baaiu, F. Couenne, Y. Le Gorrec, L. Lefèvre, and M. Tayakout. Structure-preserving infinite dimensional model reduction application to adsorption processes. Journal of Process Control, 19(3): 394-404, 2009.

Andrea Brugnoli, Daniel Alazard, Valrie Pommier-Budinger, and Denis Matignon. Port-hamiltonian formulation and symplectic discretization of plate models part i: Mindlin model for thick plates. Applied Mathematical Modelling, 75:940 - 960, 2019a. ISSN 0307-904X.

Andrea Brugnoli, Daniel Alazard, Valrie Pommier-Budinger, and Denis Matignon. Port-hamiltonian formulation and symplectic discretization of plate models part ii: Kirchhoff model for thin plates. Applied Mathematical Modelling, 75:961 - 981, 2019b. ISSN 0307-904X.

Flavio Luiz Cardoso-Ribeiro, Denis Matignon, and Laurent Lefevre. A structure-preserving partitioned finite element method for the $2 \mathrm{~d}$ wave equation. IFAC-PapersOnLine, 51(3):119-124, 2018. ISSN 2405-8963. 6th IFAC Workshop on Lagrangian and Hamiltonian Methods for Nonlinear Control LHMNC 2018.

P. David, M. Collet, and J.-M. Cote. Experimental implementation of acoustic impedance control by a 2d network of distributed smart cells. Smart Materials and Structures, 19(3):035028, 2010.

V. Duindam, A. Macchelli, S. Stramigioli, and H. eds. Bruyninckx. Modeling and Control of Complex Physical Systems - The Port-Hamiltonian Approach. Springer, Sept. 2009. ISBN 978-3-642-03195-3.

J.-F. Durand, C. Soize, and L. Gagliardini. Structural-acoustic modeling of automotive vehicles in presence of uncertainties and experimental identification and validation. Journal of the Acoustical Society of America, 124(3):Pages: 1513-1525, 2008.

Paolo Gardonio. Review of Active Techniques for Aerospace Vibro-Acoustic Control. Journal of Aircraft, 39:206-214, 2002.

G. Golo, V. Talasila, A.J. van der Schaft, and B.M. Maschke. Hamiltonian Discretization of Boundary Control Systems. Automatica, 40:757-771, 2004.

Paul Kotyczka. Finite volume structure-preserving discretization of 1d distributed-parameter porthamiltonian systems. IFAC-PapersOnLine, 49(8):298 - 303, 2016. ISSN 2405-8963. 2nd IFAC Workshop on Control of Systems Governed by Partial Differential Equations CPDE 2016. 
Paul Kotyczka and Bernhard Maschke. Discrete port-Hamiltonian formulation and numerical approximation for systems of two conservation laws. at - Automatisierungstechnik, 65(5):pp. 308-322, May 2017.

Paul Kotyczka, Bernhard Maschke, and Laurent Lefevre. Weak form of Stokes Dirac structures and geometric discretization of port-hamiltonian systems. Journal of Computational Physics, 361:442 476, 2018. ISSN 0021-9991.

Y. Le Gorrec, H. Zwart, and B. Maschke. Dirac Structures and Boundary Control Systems Associated with Skew-symmetric Differential Operators. SIAM Journal on Control and Optimization, 44:18641892, 2005.

A. Macchelli, Y. Le Gorrec, H. Ramrez, and H. Zwart. On the synthesis of boundary control laws for distributed port-hamiltonian systems. IEEE Transactions on Automatic Control, 62(4):1700-1713, 2017.

R. Moulla, L. Lefèvre, and B. Maschke. Pseudo-spectral methods for the spatial symplectic reduction of open systems of conservation laws. Journal of computational Physics, 231:1272-1292, 2011.

R. Ortega, A.J. van der Schaft, I. Mareels, and B. Maschke. Putting energy back in control. IEEE Control Systems Magazine, 21(2):18-32, April 2001.

Anass Serhani, Denis Matignon, and Ghislain Haine. Structure-preserving finite volume method for 2d linear and non-linear port-hamiltonian systems. IFAC-PapersOnLine, 51(3):131 - 136, 2018. ISSN 2405-8963. 6th IFAC Workshop on Lagrangian and Hamiltonian Methods for Nonlinear Control LHMNC 2018.

Marko Seslija, Arjan van der Schaft, and Jacquelien Scherpen. Discrete exterior geometry approach to structure-preserving discretization of distributed-parameter port-Hamiltonian systems. Journal of Geometry and Physics, 62(6):1509-1531, 2012.

V. Trenchant, H. Ramirez, Y. Le Gorrec, and P. Kotyczka. Structure preserving spatial discretization of $2 \mathrm{~d}$ hyperbolic systems using staggered grids finite difference. In 2017 American Control Conference (ACC), pages 2491-2496, May 2017a.

V. Trenchant, T. Vu, H. Ramirez, L. Lefvre, and Y. Le Gorrec. On the use of structural invariants for the distributed control of infinite dimensional port-hamitonian systems. In 2017 IEEE 56th Annual Conference on Decision and Control (CDC), pages 47-52, Dec 2017b.

Vincent Trenchant, Yassine Fares, Hector Ramirez, and Yann Le Gorrec. A port-hamiltonian formulation of a 2d boundary controlled acoustic system. IFAC-PapersOnLine, 48(13):235 - 240, 2015. ISSN 2405-8963. 5th IFAC Workshop on Lagrangian and Hamiltonian Methods for Nonlinear Control LHMNC 2015.

Vincent Trenchant, Weiwei Hu, Hector Ramirez, and Yann Le Gorrec. Structure preserving finite differences in polar coordinates for heat and wave equations. IFAC-PapersOnLine, 51(2):571 - 576, $2018 \mathrm{a}$. ISSN 2405-8963. 9th Vienna International Conference on Mathematical Modelling.

Vincent Trenchant, Hector Ramirez, Yann Le Gorrec, and Paul Kotyczka. Finite differences on staggered grids preserving the port-hamiltonian structure with application to an acoustic duct. Journal of Computational Physics, 373:673 - 697, 2018b. ISSN 0021-9991. 
A.J. van der Schaft. L $L_{2}$-gain and Passivity Techniques in Nonlinear Control. Communications and Control Engineering Series. Springer-Verlag, 2000. ISBN 9781852330736.

A.J. van der Schaft and B. Maschke. Hamiltonian Formulation of Distributed Parameter Systems with Boundary Energy Flow. Journal of Geometry and Physics, 42:166-194, 2002.

Ngoc Minh Trang Vu, Laurent Lefevre, Rmy Nouailletas, and Sylvain Bremond. Symplectic spatial integration schemes for systems of balance equations. Journal of Process Control, 51:1 - 17, 2017. ISSN 0959-1524.

Ngoc Minh Trang Vu, Laurent Lefvre, and Bernhard Maschke. Geometric spatial reduction for porthamiltonian systems. Systems \& Control Letters, 125:1 - 8, 2019. ISSN 0167-6911.

Yongxin Wu, Boussad Hamroun, Yann Le Gorrec, and Bernhard Maschke. Power preserving model reduction of $2 \mathrm{~d}$ vibro-acoustic system: A port hamiltonian approach. IFAC-PapersOnLine, 48(13): 206 - 211, 2015. ISSN 2405-8963. 5th IFAC Workshop on Lagrangian and Hamiltonian Methods for Nonlinear Control LHMNC 2015.

\section{Appendix 1: Finite dimensional Dirac structure approximation of an infinitesimal square grid}

The structure of the vibro-acoustic system (7) can be approximated by substituting the flows approximations (11), (12) and the efforts approximations (14), (15) into (7):

$$
\begin{gathered}
f_{a b c d}^{\theta} w_{a b c d}^{\theta}=-\mathrm{d}\left(e_{a b}^{p} w_{a b}^{p}+e_{b c}^{p} w_{b c}^{p}+e_{c d}^{p} w_{c d}^{p}+e_{d a}^{p} w_{d a}^{p}\right) \\
f_{a b}^{\Gamma} w_{a b}^{\Gamma}+f_{b c}^{\Gamma} w_{b c}^{\Gamma}+f_{c d}^{\Gamma} w_{c d}^{\Gamma}+f_{d a}^{\Gamma} w_{d a}^{\Gamma} \\
=\mathrm{d}\left(e^{v} w_{a}^{v}+e_{b}^{v} w_{b}^{v}+e_{c}^{v} w_{c}^{v}+e^{v} w^{v}\right)
\end{gathered}
$$

We deduce the following compatibility conditions between the two forms and one forms, one forms and between the zero-forms:

$$
\begin{aligned}
d w_{i}^{p} & =w_{a b c d}^{\theta} \quad i \in\{a b, b c, c d, d a\} \\
d w_{a}^{v} & =w_{d a}^{T}-w_{a b}^{\Gamma} ; \\
d w_{b}^{v} & =w_{a b}^{\Gamma}-w_{b c}^{\Gamma} ; \\
d w_{c}^{v} & =w_{d c}^{\Gamma}-w_{c d}^{\Gamma} ; \\
d w^{v} & =w_{c d}^{\Gamma}-w_{d a}^{\Gamma}
\end{aligned}
$$

Substituting the above compatibility conditions (68) into equations (66) and (67) and integrating over $\mathscr{Z}_{a b c d}$, the relations between the approximated flow and effort variables are given by:

$$
\begin{aligned}
& f_{a b c d}^{\theta}=-e_{a b}^{p}-e_{b c}^{p}-e_{c d}^{\mathrm{p}}-e_{d a}^{p} \\
& f_{a b}^{\Gamma}=e_{b}^{v}-e_{a}^{v}, \quad f_{b c}^{\Gamma}=e_{c}^{v}-e_{b}^{v}, \\
& f_{c d}^{\Gamma}=e_{d}^{v}-e_{c}^{v}, \quad f_{d a}^{\Gamma}=e_{a}^{v}-e_{d}^{v} .
\end{aligned}
$$

The objective here is to get a finite dimensional system with port-Hamiltonian structure guarantees the energy balance. To do so, we compute the net power over an infinitesimal square grid $a b c d P_{a b c d}^{n e t}=$ $P_{a b c d}^{\theta}+P_{a b c d}^{\Gamma}+P_{a b c d}^{B}$ composed of the kinetic power, the potential power and the power through the boundary. 
The kinetic power over an infinitesimal square grid is computed by using the approximated variables and compatibility conditions as:

$$
P_{a b c d}^{\theta}=\int_{\mathscr{L}_{a b c d}} e^{v}(t, z) \wedge f^{\theta}(t, z)=f_{a b c d}^{\theta} e_{a b c d}^{\theta}
$$

where $e_{a b c d}^{\theta}=\alpha_{a} e_{a}^{v}+\alpha_{b} e_{b}^{v}+\alpha_{c} e_{c}^{v}+\alpha_{d} e_{d}^{v}$ and

$$
\alpha_{m}:=\int_{\mathscr{Z}_{a b c d}} w_{m}^{v} \wedge w_{a b c d}^{\theta}, m \in\{a, b, c, d\} .
$$

The potential power $P_{a b c d}^{\Gamma}$ in the domain can be computed as

$$
P_{a b c d}^{\Gamma}=\int_{\mathscr{Z}_{a b c d}} e^{p}(t, z) \wedge f^{\Gamma}(t, z)=f_{a b}^{\Gamma} e_{a b}^{\Gamma}+f_{c d}^{\Gamma} e_{c d}^{\Gamma}+f_{d a}^{\Gamma} e_{d a}^{\Gamma}
$$

with the ports $\left(f_{a b}^{\Gamma}, e_{a b}^{\Gamma}\right),\left(f_{c d}^{\Gamma}, e_{c d}^{\Gamma}\right)$ and $\left(f_{d a}^{\Gamma}, e_{d a}^{\Gamma}\right)$ identified by

$$
\begin{aligned}
e_{a b}^{\Gamma}= & \left(\alpha_{b}-\beta_{b, a b}\right) e_{a b}^{p}+\left(\alpha_{b}-\beta_{b, b c}\right) e_{b c}^{p} \\
& +\left(\alpha_{b}-\beta_{b, c d}\right) e_{c d}^{p}(t)+\left(\alpha_{b}-\beta_{b, d a}\right) e_{d a}^{p} \\
e_{c d}^{\Gamma}= & \left(\beta_{c, a b}-\alpha_{c}\right) e_{a b}^{p}+\left(\beta_{c, b c}-\alpha_{c}\right) e_{b c}^{p} \\
& +\left(\beta_{c, c d}-\alpha_{c}\right) e_{c d}^{p}+\left(\beta_{c, d a}-\alpha_{c}\right) e_{d a}^{p} \\
e_{d a}^{\Gamma}= & \left(\beta_{c, a b}+\beta_{d, a b}-\alpha_{c}-\alpha_{d}\right) e_{a b}^{p} \\
& +\left(\beta_{c, b c}+\beta_{d, b c}-\alpha_{c}-\alpha_{d}\right) e_{b c}^{p} \\
& +\left(\beta_{c, c d}+\beta_{d, c d}-\alpha_{c}-\alpha_{d}\right) e_{c d}^{p} \\
& +\left(\beta_{c, d a}+\beta_{d, d a}-\alpha_{c}-\alpha_{d}\right) e_{d a}^{p}
\end{aligned}
$$

with $\alpha_{m}$ defined as (72) and

$$
\beta_{m, l}=\int_{\partial \mathscr{Z}_{a b c d}} w_{m}^{v} \wedge w_{l}^{p}, \quad \begin{aligned}
& m \in\{a, b, c, d\} \\
& l \in\{a b, b c, c d, d a\}
\end{aligned} .
$$

The power corresponding to the boundary can be computed as follows

$$
\begin{aligned}
P_{a b c d}^{B} & =\int_{\partial \mathscr{Z}_{a b c d}} e^{B}(t, z) \wedge f^{B}(t, z) \\
& =e_{a b}^{B} f_{a b}^{B}+e_{b c}^{B} f_{b c}^{B}+e_{c d}^{B} f_{c d}^{B}+e_{d a}^{B} f_{d a}^{B}
\end{aligned}
$$

by identifying the ports on the boundary as $\left(f_{a b}^{B}, e_{a b}^{B}\right),\left(f_{b c}^{B}, e_{b c}^{B}\right),\left(f_{c d}^{B}, e_{c d}^{B}\right)$ and $\left(f_{d a}^{B}, e_{d a}^{B}\right)$, where

$$
\begin{aligned}
f_{a b}^{B} & =\beta_{a, a b} e_{a}^{v}+\beta_{b, a b} e_{b}^{v}+\beta_{c, a b} e_{c}^{v}+\beta_{d, a b} e_{d}^{v} \\
f_{b c}^{B} & =\beta_{a, b c} e_{a}^{v}+\beta_{b, b c} e_{b}^{v}+\beta_{c, b c} e_{c}^{v}+\beta_{d, b c} e_{d}^{v} \\
f_{c d}^{B} & =\beta_{a, c d} e_{a}^{v}+\beta_{b, c d} e_{b}^{v}+\beta_{c, c d} e_{c}^{v}+\beta_{d, c d} e_{d}^{v} \\
f_{d a}^{B} & =\beta_{a, d a} e_{a}^{v}+\beta_{b, d a} e_{b}^{v}+\beta_{c, d a} e_{c}^{v}+\beta_{d, d a} e_{d}^{v}
\end{aligned}
$$

with $\beta_{m, l}=\int_{\partial \mathscr{Z}_{a b c d}} w_{m}^{v} \wedge w_{l}^{p}, m \in\{a, b, c, d\}$ and $l \in\{a b, b c, c d, d a\}$.

Using the net power relations (71), (73) and (75), we can write the finite dimensional Dirac structure over each infinitesimal square grid with its image representation [Duindam et al., 2009] as follows

$$
\mathscr{D}_{a b c d}=\left\{\left(f_{a b c d}, e_{a b c d}\right) \mid \begin{array}{l}
f_{a b c d}=E_{a b c d}^{*} \lambda_{a b c d} \\
e_{a b c d}=F_{a b c d}^{*} \lambda_{a b c d}
\end{array}, \lambda_{a b c d} \in \mathbb{R}^{8}\right\}
$$


where

$$
\begin{gathered}
f_{a b c d}=\left[f_{a b c d}^{\theta}, f_{a b}^{\Gamma}, f_{c d}^{\Gamma}, f_{d a}^{\Gamma}, f_{a b}^{B}, f_{b c}^{B}, f_{c d}^{B}, f_{d a}^{B}\right]^{T} \in \mathbb{R}^{8} \\
e_{a b c d}=\left[e_{a b c d}^{\theta}, e_{a b}^{\Gamma}, e_{c d}^{\Gamma}, e_{d a}^{\Gamma}, e_{a b}^{B},-e_{b c}^{B},-e_{c d}^{B}, e_{d a}^{B}\right]^{T} \in \mathbb{R}^{8} \\
\lambda_{a b c d}=\left[e_{a}^{v}, e_{b}^{v}, e_{c}^{v}, e_{d}^{v}, e_{a b}^{p}, e_{b c}^{p}, e_{c d}^{p}, e_{d a}^{p}\right]^{T} \in \mathbb{R}^{8}
\end{gathered}
$$

and the matrices $E_{a b c d}$ and $F_{a b c d}$ are define by

$$
\begin{aligned}
E_{a b c d}^{*} & =\left[\begin{array}{cccccccc}
0 & 0 & 0 & 0 & -1 & -1 & -1 & -1 \\
-1 & 1 & 0 & 0 & 0 & 0 & 0 & 0 \\
0 & 0 & -1 & 1 & 0 & 0 & 0 & 0 \\
1 & 0 & 0 & -1 & 0 & 0 & 0 & 0 \\
\beta_{a, a b} & \beta_{b, a b} & \beta_{c, a b} & \beta_{d, a b} & 0 & 0 & 0 & 0 \\
\beta_{a, b c} & \beta_{b, b c} & \beta_{c, b c} & \beta_{d, b c} & 0 & 0 & 0 & 0 \\
\beta_{a, c d} & \beta_{b, c d} & \beta_{c, c d} & \beta_{d, c d} & 0 & 0 & 0 & 0 \\
\beta_{a, d a} & \beta_{b, d a} & \beta_{c, d a} & \beta_{d, d a} & 0 & 0 & 0 & 0
\end{array}\right] \\
F_{a b c d}^{*}= & {\left[\begin{array}{cccccccc}
\alpha_{a} & \alpha_{b} & \alpha_{c} & \alpha_{d} & 0 & 0 & 0 & 0 \\
0 & 0 & 0 & 0 & \gamma_{b, a b} & \gamma_{b, b c} & \gamma_{b, c d} & \gamma_{b, d a} \\
0 & 0 & 0 & 0 & -\gamma_{c, a b} & -\gamma_{c, b c} & -\gamma_{c, c d} & -\gamma_{c, d a} \\
0 & 0 & 0 & 0 & \gamma_{a b} & \gamma_{b c} & \gamma_{c d} & \gamma_{d a} \\
0 & 0 & 0 & 0 & 1 & 0 & 0 & 0 \\
0 & 0 & 0 & 0 & 0 & 1 & 0 & 0 \\
0 & 0 & 0 & 0 & 0 & 0 & 1 & 0 \\
0 & 0 & 0 & 0 & 0 & 0 & 0 & 1
\end{array}\right] }
\end{aligned}
$$

with $\gamma_{m, l}=\alpha_{m}-\beta_{m, l}, \gamma_{l}=\beta_{l}-\alpha_{c d}, \alpha_{c d}=\alpha_{c}+\alpha_{d}, \beta_{l}=\beta_{c, l}+\beta_{d, l}, m \in\{a, b, c, d\}$, and $l \in\{a b, b c, c d, d a\}$.

Using the weighting function (17)-(19), the parameters $\alpha$ and $\beta$ can be computed as follows:

$$
\begin{aligned}
\alpha_{a, a b c d} & =\int_{z_{a b c d}} w_{a} \wedge w_{a b c d} \\
& =\int_{y_{1}}^{y_{2}} \int_{x_{1}}^{x_{2}} \frac{\left(x_{2}-x\right)}{\left(x_{2}-x_{1}\right)} \frac{\left(y_{2}-y\right)}{\left(y_{2}-y_{1}\right)} \frac{1}{\left(x_{2}-x_{1}\right)\left(y_{2}-y_{1}\right)} d x \wedge d y \\
& =\frac{1}{4} .
\end{aligned}
$$

By doing the same computation, one can find $\alpha_{a, a b c d}=\alpha_{b, a b c d}=\alpha_{c, a b c d}=\alpha_{d, a b c d}=\frac{1}{4}$ and

$$
\begin{aligned}
\beta_{a, a b} & =\int_{\partial z_{a b c d}} w_{a} \wedge w_{a b} \\
& =\left.\int_{x_{1}}^{x_{2}} \frac{\left(x_{2}-x\right)}{\left(x_{2}-x_{1}\right)} \frac{\left(y_{2}-y\right)}{\left(y_{2}-y_{1}\right)} \frac{\left(y_{2}-y\right)}{\left(x_{2}-x_{1}\right)\left(y_{2}-y_{1}\right)} d x\right|_{y=y_{1}} \\
& =\frac{1}{2} .
\end{aligned}
$$

In the same way:

$$
\begin{array}{llll}
\beta_{a, a b}=\frac{1}{2}, & \beta_{b, a b}=\frac{1}{2}, & \beta_{c, a b}=0, & \beta_{d, a b}=0, \\
\beta_{a, b c}=0, & \beta_{b, b c}=\frac{1}{2}, & \beta_{c, b c}=\frac{1}{2}, & \beta_{d, b c}=0, \\
\beta_{a, c d}=0, & \beta_{b, c d}=0, & \beta_{c, c d}=\frac{1}{2}, & \beta_{d, c d}=\frac{1}{2}, \\
\beta_{a, d a}=\frac{1}{2}, & \beta_{b, d a}=0, & \beta_{c, d a}=0, & \beta_{d, d a}=\frac{1}{2} .
\end{array}
$$


Furthermore, the following parameters can be computed:

$$
\begin{aligned}
& \alpha_{c d}=\alpha_{c, a b c d}+\alpha_{d, a b c d}=\frac{1}{2} \\
& \beta_{a b}=\beta_{c, a b}+\beta_{d, a b}=0 \\
& \beta_{b c}=\beta_{c, b c}+\beta_{d, b c}=\frac{1}{2} \\
& \beta_{c d}=\beta_{c, c d}+\beta_{d, c d}=1 \\
& \beta_{d a}=\beta_{c, d a}+\beta_{d, d a}=\frac{1}{2}
\end{aligned}
$$

Using the above coefficients, one can get the finite dimenaionla Dirac structure approximation of the infinitesimal square grid (20).

\section{Appendix 2: Constitutive equations approximation}

To construct the explicit finite dimensional approximation of the port-Hamiltonian system on the infinitesimal square grid, we should also derive the approximation of the constitutive relations (9). Now let consider the approximation of the energy variables as:

$$
\begin{aligned}
\theta(t, z) & =\theta_{a b c d} w_{a b c d}^{\theta} \\
\Gamma(t, z) & =\Gamma_{a b} w_{a b}^{\Gamma}+\Gamma_{b c} w_{b c}^{\Gamma}+\Gamma_{c d} w_{c d}^{\Gamma}+\Gamma_{d a} w_{d a}^{\Gamma}
\end{aligned}
$$

In order to derive the constitutive equations of the finite dimensional approximation, we compute the energy over an infinitesimal square grid with the approximated energy variables. First, we consider the kinetic energy over the infinitesimal square $\mathscr{Z}_{a b c d}$ as

$$
\begin{aligned}
H_{a b c d}^{\theta} & =\frac{1}{2} \int_{\mathscr{Z}_{a b c d}} \theta(z, t) \wedge * \frac{\theta(z, t)}{\rho_{0}} \\
& =\frac{\theta_{a b c d}^{2}}{2} \int_{\mathscr{Z}_{a b c d}} \frac{w_{a b c d}^{\theta} \wedge * w_{a b c d}^{\theta}}{\rho_{0}}=\frac{\theta_{a b c d}^{2}}{2 M}
\end{aligned}
$$

with $M^{-1}=\int_{\mathscr{Z}_{a b c d}} \frac{w_{a b c d}^{\theta} \wedge * w_{a b c d}^{\theta}}{\rho_{0}}$. Then using the weighting function (17), one can get:

$$
\begin{aligned}
M^{-1} & =\int_{\mathscr{Z}_{a b c d}} \frac{w_{a b c d}^{\theta} \wedge * w_{a b c d}^{\theta}}{\rho_{0}} \\
& =\frac{1}{\rho_{0}} \int_{y_{1}}^{y_{2}} \int_{x_{1}}^{x_{2}}\left[\frac{1}{\left(x_{2}-x_{1}\right)\left(y_{2}-y_{1}\right)}\right]^{2} d x \wedge d y \\
& =\frac{1}{\rho_{0}} \frac{1}{\left(x_{2}-x_{1}\right)\left(y_{2}-y_{1}\right)}=\frac{1}{\rho_{0}} \frac{1}{R_{\text {inf }} L_{i n f}}
\end{aligned}
$$

where $L_{i n}=x_{2}-x_{1}$ and $R_{i n}=y_{2}-y_{1}$ are the length and the width of the infinitesimal square grid respectively.

Before deriving the potential energy over the square $\mathscr{Z}_{a b c d}$, we rewrite the volumetric expansion by using the compatibility conditions (68) as:

$$
\Gamma(t, z)=\Gamma_{a b} d w_{b}^{v}-\Gamma_{c d} d w_{c}^{v}-\Gamma_{d a}\left(d w_{c}^{v}+d w_{d}^{v}\right) .
$$

These finite dimensional energy variables has been chosen with respect to the effort variables over the square from the net power balance (73). Using the energy variables defined above, the potential energy is expressed as:

$$
\begin{aligned}
H_{a b c d}^{\Gamma} & =\frac{1}{2} \int_{\mathscr{Z}_{a b c d}} \Gamma(z, t) \wedge * \frac{\Gamma(z, t)}{\chi_{s}} \\
& =\frac{1}{2}\left[\frac{\Gamma_{a b}^{2}}{\chi_{s 1}}+\frac{\Gamma_{c d}^{2}}{\chi_{s 2}}+\frac{\Gamma_{d a}^{2}}{\chi_{s 3}}-\frac{2 \Gamma_{a b} \Gamma_{c d}}{\chi_{s 4}}-\frac{2 \Gamma_{a b} \Gamma_{d a}}{\chi_{s 5}}+\frac{2 \Gamma_{c d} \Gamma_{d a}}{\chi_{s 6}}\right]
\end{aligned}
$$


with $\chi_{s 1}^{-1}=\int_{\mathscr{Z}_{a b c d}} \frac{d w_{b}^{v} \wedge * d w_{b}^{v}}{\chi_{s}}, \chi_{s 2}^{-1}=\int_{\mathscr{Z}_{a b c d}} \frac{d w_{c}^{v} \wedge * d w_{c}^{v}}{\chi_{s}}, \chi_{s 3}^{-1}=\int_{\mathscr{Z}_{a b c d}} \frac{\left(d w_{c}^{v}+d w_{d}^{v}\right) \wedge *\left(d w_{c}^{v}+d w_{d}^{v}\right)}{\chi_{s}}, \chi_{s 4}^{-1}=\int_{\mathscr{Z}_{a b c d}} \frac{d w_{b}^{v} \wedge * d w_{c}^{v}+d w_{c}^{v} \wedge * d w_{b}^{v}}{2 \chi_{s}}$, $\chi_{s 5}^{-1}=\int_{\mathscr{Z}_{a b c d}} \frac{d w_{b}^{v} \wedge *\left(d w_{c}^{v}+d w_{d}^{v}\right)+\left(d w_{c}^{v}+d w_{d}^{v}\right) \wedge * d w_{b}^{v}}{2 \chi_{s}}, \chi_{s 6}^{-1}=\int_{\mathscr{Z}_{a b c d}} \frac{d w_{c}^{v} \wedge *\left(d w_{c}^{v}+d w_{d}^{v}\right)+\left(d w_{c}^{v}+d w_{d}^{v}\right) \wedge * d w_{c}^{v}}{2 \chi_{s}}$. Using the chosen weighting functions (19), we can compute the above parameters:

$$
\begin{aligned}
& \chi_{s 1}^{-1}=\frac{1}{3 \chi_{s}}\left[\frac{y_{2}-y_{1}}{x_{2}-x_{1}}+\frac{x_{2}-x_{1}}{y_{2}-y_{1}}\right]=\frac{1}{3 \chi_{s}}\left[\frac{R_{i n}}{L_{i n}}+\frac{L_{i n}}{R_{i n}}\right], \\
& \chi_{s 2}^{-1}=\frac{1}{3 \chi_{s}}\left[\frac{y_{2}-y_{1}}{x_{2}-x_{1}}+\frac{x_{2}-x_{1}}{y_{2}-y_{1}}\right]=\frac{1}{3 \chi_{s}}\left[\frac{R_{i n}}{L_{i n}}+\frac{L_{i n}}{R_{i n}}\right], \\
& \chi_{s 3}^{-1}=\frac{1}{\chi_{s}} \frac{x_{2}-x_{1}}{y_{2}-y_{1}}=\frac{1}{\chi_{s}} \frac{L_{i n}}{R_{i n}} \\
& \chi_{s 4}^{-1}=\frac{1}{3 \chi_{s}}\left[\frac{y_{2}-y_{1}}{x_{2}-x_{1}}+\frac{x_{2}-x_{1}}{y_{2}-y_{1}}\right]=\frac{1}{3 \chi_{s}}\left[\frac{R_{i n}}{L_{i n}}+\frac{L_{i n}}{R_{i n}}\right], \\
& \chi_{s 5}^{-1}=\frac{1}{6 \chi_{s}} \frac{x_{2}-x_{1}}{y_{2}-y_{1}}=\frac{1}{6 \chi_{s}} \frac{L_{i n}}{R_{i n}} \\
& \chi_{s 6}^{-1}=\frac{1}{2 \chi_{s}} \frac{x_{2}-x_{1}}{y_{2}-y_{1}}=\frac{1}{2 \chi_{s}} \frac{L_{i n}}{R_{i n}} .
\end{aligned}
$$

The finite dimensional approximation of the constitutive relations can be written as

$$
\left[\begin{array}{c}
e_{a b c d}^{\theta} \\
e_{a b}^{\Gamma} \\
e_{c d}^{\Gamma} \\
e_{d a}^{\Gamma}
\end{array}\right]=Q_{a b c d}\left[\begin{array}{c}
\theta_{a b c d} \\
\Gamma_{a b} \\
\Gamma_{c d} \\
\Gamma_{d a}
\end{array}\right]
$$

with

$$
Q_{a b c d}=\left[\begin{array}{cccc}
S_{i n} \frac{1}{\rho_{0}} & 0 & 0 & 0 \\
0 & \frac{1}{3} S_{2 i n} \frac{1}{\chi_{s}} & -\frac{1}{3} S_{2 i n} \frac{1}{\chi_{s}} & -S_{1 \text { in }} \frac{1}{6 \chi_{s}} \\
0 & -\frac{1}{3} S_{2 i n} \frac{1}{\chi_{s}} & \frac{1}{3} S_{2 i n} \frac{1}{\chi_{s}} & S_{1 \text { in }} \frac{1}{2 \chi_{s}} \\
0 & -S_{1 i n} \frac{1}{6 \chi_{s}} & S_{1 i n} \frac{1}{2 \chi_{s}} & S_{1 i n} \frac{1}{\chi_{s}}
\end{array}\right] .
$$

and $S_{i n}=\frac{1}{L_{i n} R_{i n}}, S_{1 i n}=\frac{R_{i n}}{L_{i n}}$ and $S_{2 i n}=\frac{R_{i n}}{L_{i n}}+\frac{L_{i n}}{R_{i n}}$. 